\title{
PRELIMINARY DESIGN OF SATELLITE CONSTELLATIONS FOR A BRAZILIAN REGIONAL POSITIONING SYSTEM BY MEANS OF AN EVOLUTIONARY
} ALGORITHM

\author{
Roberto Luiz Galski \\ CRC/INPE, Av. Astronautas 1758, São José Campos, SP, Brazil, (55)12-3208-6386, \\ galski@ccs.inpe.br
}

\begin{abstract}
This paper presents and discuss some possibilities concerning to the design of a Regional Positioning System - RPS satellite constellation to cover the Brazilian territory. The paper presents an overview of constellation design possibilities for a hypothetical Brazilian Regional Positioning System. Since Brazil is located near the equator plane, the idea is to think about a RPS satellite constellation that takes advantage of this fact in order to design a cost effective regional system that aims at covering primarily only the Brazilian territory. In this sense, three satellite constellation types were considered. The first one is based only on geosynchronous satellites. The second one is based on Medium Earth Orbit - MEO satellites placed in the equator plane or near to it, and the third one is composed by Low Earth Orbit - LEO satellites with low to moderate orbital plane inclination angles. A preliminary optimization process was run in order to obtain an initial guess of a good design. The obtained design constellations are presented and discussed.
\end{abstract}

Keywords: satellite positioning system, satellite constellation design, optimization

\section{Introduction}

Global Navigation Satellite Systems - GNSS provide autonomous geo-spatial positioning with global coverage. They allow portable receivers located on the ground or near earth's surface to determine their location (longitude, latitude, and altitude) to within a few meters using position and time signals transmitted by radio from satellites. Although originally developed for military purposes, nowadays GNSS are a dual-use technology, since they have significant military and civilian applications. They have become a widely used and a useful tool for a large variety of applications in a great number of fields such as, for example, commerce, science and technology, tracking and surveillance. Accurate timing provided by such systems facilitates everyday activities such as banking, mobile phone operations, and even the control of power grids. Around the entire world, professionals from many different areas perform their work more efficiently, safely, economically and accurately. In this way, GNSS are a crucial component of the global information infrastructure [1].

Today, the existing GNSS constellations like the American GPS [2] or the Russian GLONASS [3] systems are continuously being updated with new and improved satellites providing new signals, new frequencies, and new functionalities. In addition, other GNSS and augmentation systems are in the process of being deployed, such as the European Galileo satellite system, Japan's Quasi-Zenith satellite system, India's Regional Navigational Satellite System - IRNSS, or China's Compass satellite system [4].

In [5], the concept of a Regional Positioning System based on a Low Earth Orbit satellite constellation is presented and discussed. In the case of the mentioned study, the aim of the proposed constellation was to provide positioning coverage for a region of about $3,000 \mathrm{~km}$ around a specific point on the globe, with coordinates $122^{\circ} \mathrm{E}$ and $25^{\circ} \mathrm{N}$. The metric used to assess the goodness of the proposed constellation solution was the Geometric Dilution of Precision - GDOP [6] calculated for several points inside the region covered. The results reported that the aim was achieved with a 
satellite constellation composed by 75 satellites disposed in four orbital planes with an altitude of about $800 \mathrm{~km}$ and circular orbits.

Considering the importance of a satellite positioning system for countries that possess vast territories, it is reasonable to believe that Brazil will develop its own system. In this way, this paper presents and discuss some possibilities concerning to aspects of designing satellite constellations for a Regional Positioning System - RPS with the objective of covering the Brazilian territory. In other words, it presents an overview of constellation design possibilities for a hypothetical Brazilian Regional Positioning System. Since Brazil is located near the Equator line, this fact was taken into account in order to design a cost effective regional system that aims at covering primarily only the Brazilian territory. In this sense, three satellite constellation types were considered. The first one is composed by Low Earth Orbit - LEO satellites with low to moderate orbital plane inclination angles. The second one is based on Medium Earth Orbit - MEO satellites placed near the equator plane, and. the third one is based only on geosynchronous satellites. A preliminary optimization process was run in order to obtain improved designs for each constellation type. The word preliminary is used here to express that, as the computation time allowed for the process was relatively short, the presented design must be seen with a grain of salt, since it is known elsewhere that when dealing with nonlinear optimization problems and stochastic optimization algorithms as in this case, it is impossible to guarantee that the absolute best design was found.

In this work, one uses the GDOP as a metric for the evaluation of the constellation candidate solutions during the optimization procedure. The optimization task is performed by a hybrid evolutionary algorithm. The GEO + ES algorithm [7] was developed in order to conjugate the good convergence properties of the Generalized Extremal Optimization - GEO [8] algorithm with the self-tuning characteristics present in the Evolution Strategies - ES methods [9]. Describing both algorithms a little further, ES is a well known technique, whose first developments remounts back to the early 1960's and whose posterior versions were among the first algorithms to include selftuning (of their internal parameters) as a feature and it is an Evolutive Algorithm (EA) [9]. GEO, by its turn, is a global search meta-heuristic, based on a model of natural evolution [10], and specially devised to be used in complex optimization problems and has been successfully applied to a broad variety of science and engineering real-world problems [11-13]. In [7], four improvements for the canonical GEO algorithm were suggested. One of them, called $\mathrm{GEO}_{4}$, uses real coded variables instead of the binary ones used by the canonical GEO. It showed excellent performance results with test functions, in terms of convergence properties, and it was subsequently used to form the GEO + ES hybrid.

The paper is organized as follows: Section 2 gives information about the kinds of constellation one wants to design and the optimization strategy used. In Section 3, the results are presented and commented. In Section 4, the conclusions are presented.

\section{Optimization Strategy}

The optimization strategy adopted is the same for all three constellations types considered. It consists in optimizing the average Geometric Dilution of Precision - GDOP [6] occurred for a set of 5 hypothetical receivers placed on the Brazilian territory. Figure 1 gives the location of the 5 receivers and the corresponding visibility circles for a Low Earth Orbit $(\sim 1000 \mathrm{~km})$ and a minimum elevation angle of $10^{\circ}$. Their location was defined in order to be representative of the whole Brazilian territory. In order to calculate the GDOP average, the orbit of all satellites in the constellation is numerically propagated using mathematical models that include appropriate orbit perturbations for each orbit type. The GDOP of each receiver is calculated over a simulation period previously chosen and with a predefined time step, resulting in a set of GDOP values covering the 
whole propagation interval. The average GDOP is calculated considering the whole set of data retrieved from all the receivers considered. The GDOP calculation procedure implemented and used in this article always uses the best four visible satellites and was validated with the help of the STK 8.1 software [14]. For that aim, the GDOP values for three different sets of 8 satellites with orbital elements arbitrarily chosen were calculated using STK and the article's procedure. The GDOP results for the test sets $\{1,2$ and 3$\}$ were $\{6.30,2.90$, and 2.92\} for STK software and $\{7.12,2.49$, and 2.54$\}$ for the article's procedure.

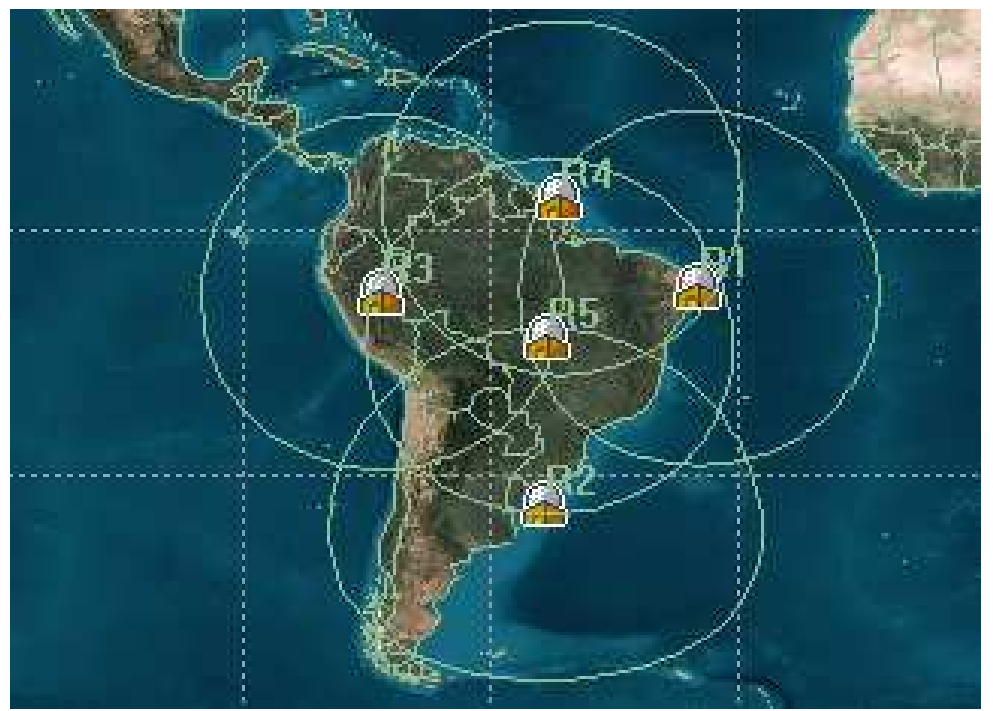

Figure 1. Location of the 5 receivers considered for the optimization.

The satellites orbit simulation, with the state vector propagation, is carried out by a computer program developed specifically to the orbit propagation of multiple satellites. It is based on the orbital dynamics routines (in Fortran) developed at INPE by the orbital dynamics group for the propagation of just one satellite. For the Low Earth Orbit constellation the propagation is done considering only the effect of the Earth's gravitational field modeled by a central force field (mass point) plus the second zonal harmonic, J2, which models the poles flatness. For the medium and geosynchronous constellation orbits the propagation is done considering also the gravitational effects of the sun and the moon and the effect of the sun radiation pressure.

For each of the three constellations some of the keplerian orbital elements at the beginning of the propagation were set as constants and some were defined as design variables of the optimization problem. In this way, it is the task of the optimization algorithm to search for the design variable values that result in the minimum value of the average GDOP. In the following, the design variables and the constant keplerian elements are defined for each constellation. The epoch considered for all the simulations was November $1^{\text {st }} 2010$ at $0 \mathrm{~h} 0 \mathrm{~min} 0 \mathrm{~s}$.

Constellation 1 (Low Earth Orbit): The orbital parameters of the constellation satellites that were considered as optimization variables are the inclination, the right ascension of the ascending node and the mean anomaly. The remaining keplerian elements were set constant to all satellites: -semimajor axis $(7,378.139 \mathrm{~km})$; -eccentricity $(0.0)$; -perigee argument $\left(0.0^{\circ}\right)$. The value defined to the semi-major axis corresponds to an orbit altitude of $1000 \mathrm{~km}$. The limits used for the design variables were $0^{\circ}$ to $35^{\circ}$ to the inclination (I) and $0^{\circ}$ to $360^{\circ}$ to the right ascension $(\Omega)$ and the mean anomaly (M).

Constellation 2 (Medium Earth Orbit): The orbital parameters that were considered as optimization variables are the inclination, the right ascension of the ascending node and the mean anomaly. The remaining keplerian elements were set as constants to all satellites: -semi-major axis 
$(26,378.139 \mathrm{~km})$; -eccentricity $(0.0)$; -perigee argument $\left(0.0^{\circ}\right)$. The value defined to the semi-major axis corresponds to an orbit altitude of $20,000 \mathrm{~km}$. The limits used for the design variables were $0^{\circ}$ to $35^{\circ}$ to the inclination (I) and $0^{\circ}$ to $360^{\circ}$ to the right ascension $(\Omega)$ and the mean anomaly (M).

Constellation 3 (Geosynchronous orbit): The orbital parameters of the constellation satellites that were considered as optimization variables are the inclination, the right ascension of the ascending node and the mean anomaly. The remaining keplerian elements were set as constants to all satellites: -semi-major axis $(42,164.139 \mathrm{~km})$; -eccentricity $(0.0)$; -perigee argument $\left(0.0^{\circ}\right)$. The value defined to the semi-major axis corresponds to the geosynchronous orbit altitude of $35,786 \mathrm{~km}$. The limits used for the design variables were $0^{\circ}$ to $60^{\circ}$ to the inclination (I) and $0^{\circ}$ to $360^{\circ}$ to the right ascension $(\Omega)$ and the mean anomaly $(\mathrm{M})$.

For Constellations 1 and 2, the optimization was run for three different numbers of satellites in the constellation. For Constellation 1, \{40;60; and 80 $\}$ satellites were considered and $\{10 ; 12$; and 14$\}$ satellites were considered for Constellation 2. For Constellation 3, only 4 satellites were considered.

\section{Results}

The GEO + ES algorithm was run for each Constellation and for each number of satellites described in the previous section. For each run, a previously defined number of generations were allowed to occur within the GEO + ES algorithm and this limit was used as a stopping criterion. For Constellations 2 and 3, it was used 500 generations, while for the more time consuming Constellation 1, 200 generations was used as limit for the search. Three mutations per variable were used, so $l_{\mathrm{j}}=l=3$ and the number of design variables was $\mathrm{L}=3 * \mathrm{NSAT}$, for Constellations 1 and 2 , where NSAT is the number of satellites in the constellation and $\mathrm{L}=2 * 4=8$, for Constellation 3 . The limits for varying $b$ were set to $b_{\text {MIN }}=1.05$ and $b_{\text {MAX }}=10$. The values of $\delta=0.0$ and of $\alpha=0.3$ were used. The four parameters just mentioned are internal parameters for GEO + ES. For those moments, during the satellite orbit propagation steps, when there were less than 4 satellites visible to a receiver, the value $10^{5}$ was arbitrarily imposed as being the GDOP of the respective receiver. The simulation period was set to one day (86400s) to all the three constellations. The time step used for the orbit propagation was 150 seconds for Constellations 1 and 2 and 30 seconds for Constellation 3.

\section{1. - Results for Constellation 1 (Low Earth Orbit - 1,000km)}

The best average GDOP values found for Constellation 1 were $\{27,145.0 ; 13,824.6$; and 11,150.1 $\}$ for $\{40 ; 80$; and 120$\}$ satellites respectively. From the GDOP values, it is possible to conclude that 40, 80 and even 120 satellites were not enough to achieve a good coverage for all the 5 receivers considered. Figures 2 to 7 give the GDOP values occurred for the 5 receivers during the propagation interval of the best solutions found for 40, 80 and 120 satellites of Constellation 1.

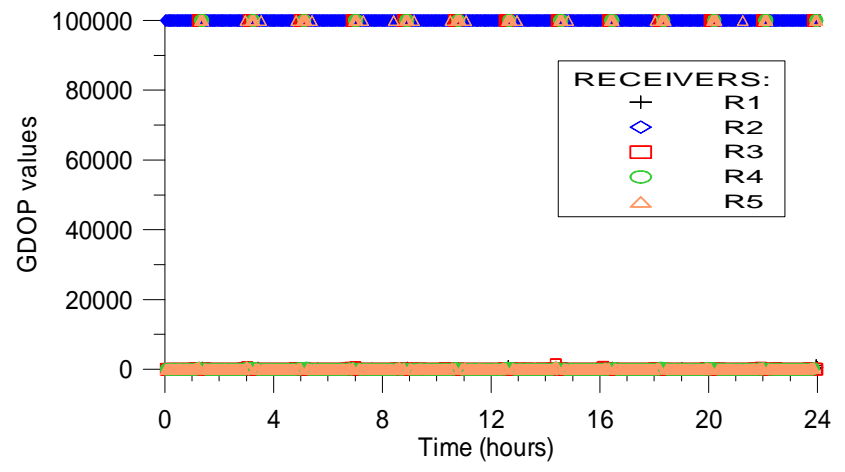

Figure 2. GDOP for Constellation 1 (40 satellites)

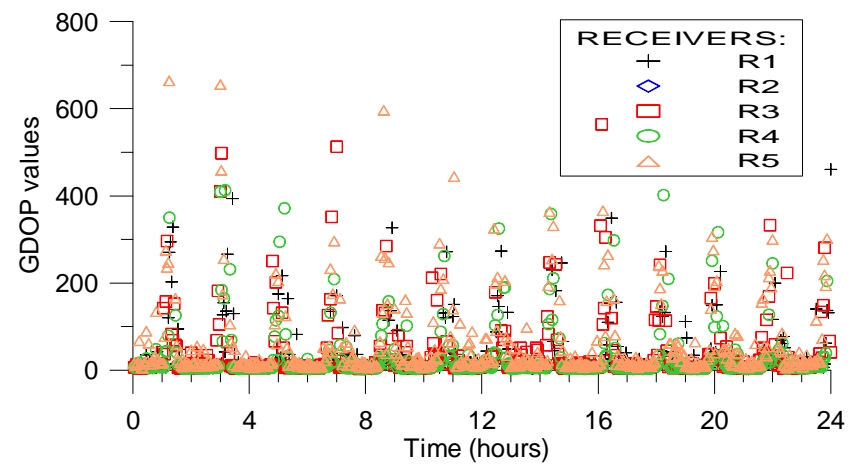

Figure 3. Zoom of Fig. 2. 
Figures 2 and 3 show that with 40 satellites receiver R2 remains practically all the time not covered by at least 4 satellites $\left(\mathrm{GDOP}=10^{5}\right)$ and the same happening to the others from time to time. Fig. 3 also shows that even when there are 4 satellites visible to the receivers the GDOP values for all the receivers are not good quite frequently. In order to quantify it numerically, from the data files it was possible to count the number of points on which GDOP is less than 10, for each receiver. Presenting it as percentage of the time interval, one has $\{53.3 \% ; 0.0 \% ; 50.7 \% ; 61.8 \% ; 15.6 \%\}$ as result for receivers $\{R 1, R 2, R 3, R 4$, and $R 5\}$ respectively.

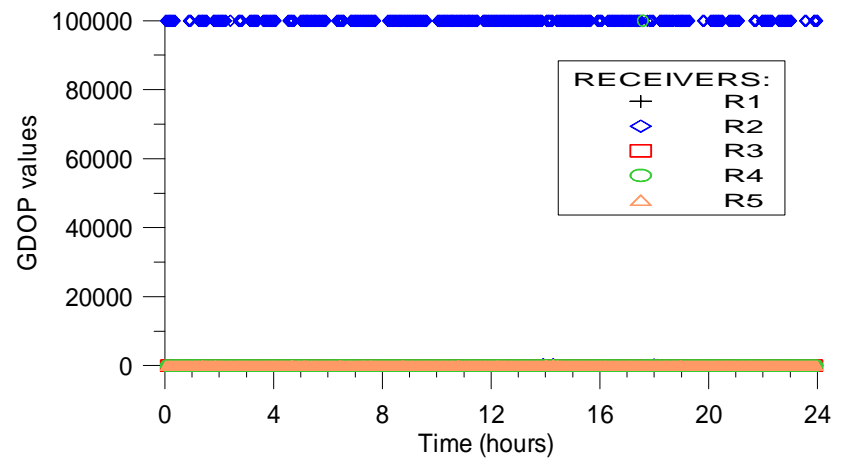

Figure 4. GDOP for Constellation 1 (80 satellites)

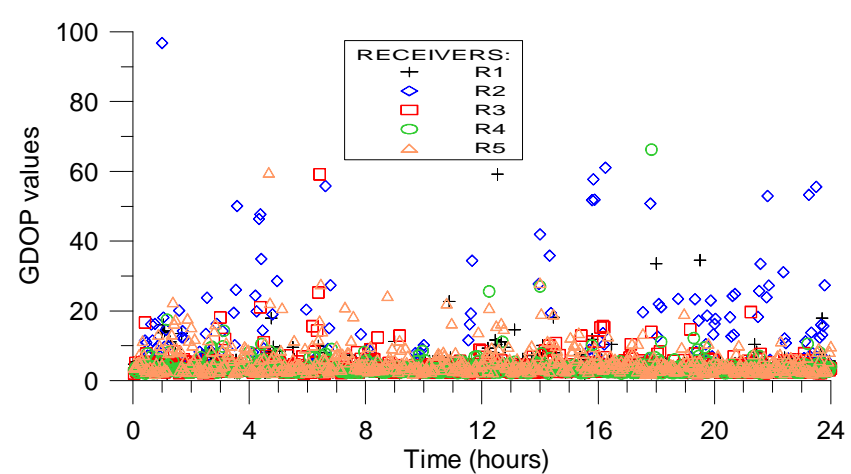

Figure 5. Zoom of Fig. 4.

Figures 4 and 5 show that with 80 satellites receiver R2 still remains most of the time not covered by at least 4 satellites while the others are covered, with only one exception for R4 at 17.6 hours. Figure 5 shows that GDOP values for all the receivers but R2 remain below 10 most of the time. In more quantitative terms, the percentages of the time interval on which GDOP was less than 10 were $\{96.4 \% ; 15.5 \% ; 96.0 \% ; 100.0 \% ; 89.6 \%\}$ for receivers $\{\mathrm{R} 1, \mathrm{R} 2, \mathrm{R} 3, \mathrm{R} 4$, and R5 $\}$ respectively.

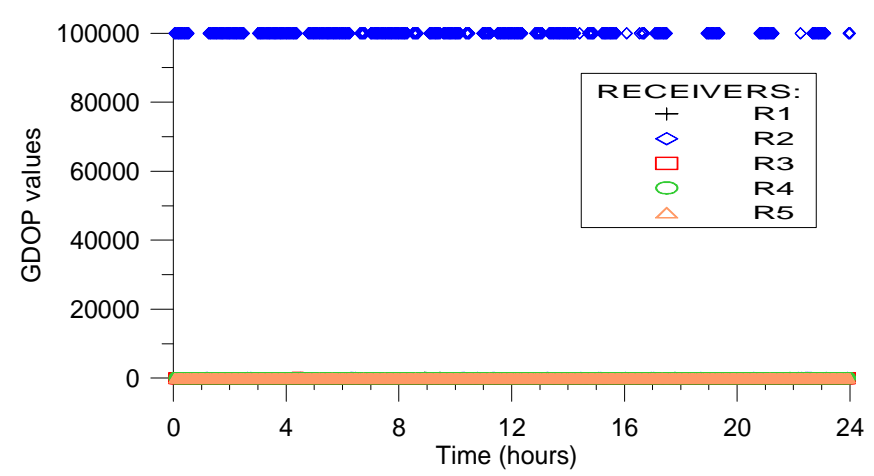

Figure 6. GDOP for Constellation 1 (120 satellites)

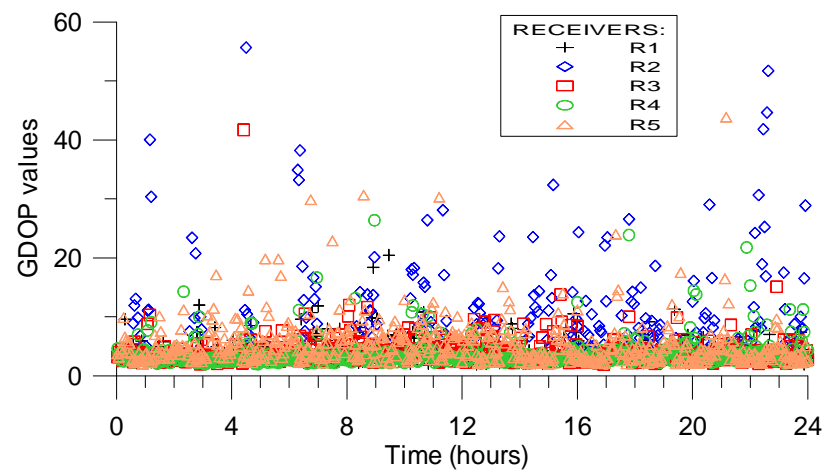

Figure 7. Zoom of Fig. 6.

Figures 6 and 7 show that with 120 satellites receiver R2 still remains most of the time not covered by at least 4 satellites while the others are covered all the time. The percentages of the time interval on which GDOP was less than 10 were $\{98.6 \% ; 29.7 \% ; 98.4 \% ; 100.0 \%$; and $93.2 \%\}$ for the receivers $\{R 1, R 2, R 3, R 4$, and $R 5\}$ respectively. The percentage results have shown a pattern, with receivers $\mathrm{R} 2$ and $\mathrm{R} 5$, in this order, as the most difficult ones to get covered by the constellations of type 1. In terms of computational time for Constellation 1 , they were $0.87,3.68$, and 6.23 days for 40, 80 and 120 satellites, respectively, on a Intel Core 2 Quad 2.83GHz PC with 2GB RAM.

\section{2. - Results for Constellation 2 (Medium Earth Orbit - 20,000km)}

The best average GDOP values found for Constellation 2 were $\{27,055.4 ; 6.87$; and 3.44$\}$ for $\{10$, 12 , and 14\} satellites respectively. From the GDOP values, it is possible to conclude that 10 satellites were not enough to achieve a good coverage for all the 5 receivers considered. In the case 
of 12 and 14 satellites, both achieved good average GDOP values. Table 1 gives the solution found for Constellation 2 with 14 satellites, where all values are in degrees.

Table 1. - Constellation 2 solution (14 satellites, GDOP = 3.44 )

\begin{tabular}{|c|c|c|}
\hline $\mathrm{I}_{1 . .14}$ & $\Omega_{1 . .14}$ & $\mathrm{M}_{1 . .14}$ \\
\hline $\begin{array}{lllll}29.6 & 33.8 & 25.5 & 1.9 & 34.9\end{array}$ & $\begin{array}{lllll}192.1 & 199.1 & 314.9 & 60.3 & 94.3\end{array}$ & $\begin{array}{lllll}184.2 & 304.8 & 8.3 & 159.8 & 255.4\end{array}$ \\
\hline $\begin{array}{lllll}11.2 & 27.0 & 35.0 & 34.0 & 35.0\end{array}$ & $\begin{array}{lllll}131.2 & 186.2 & 358.3 & 299.0 & 177.7\end{array}$ & $\begin{array}{lllll}322.2 & 124.2 & 76.0 & 191.9 & 11.8\end{array}$ \\
\hline $\begin{array}{llll}34.9 & 0.1 & 27.0 & 0.1\end{array}$ & $\begin{array}{llll}164.8 & 225.1 & 307.7 & 256.2\end{array}$ & $\begin{array}{llll}89.4 & 182.0 & 230.6 & 24.9\end{array}$ \\
\hline
\end{tabular}

Figures 8 to 13 give the GDOP values occurred for the 5 receivers during the propagation interval of the best solutions found for 10,12 and 14 satellites of Constellation 2 .

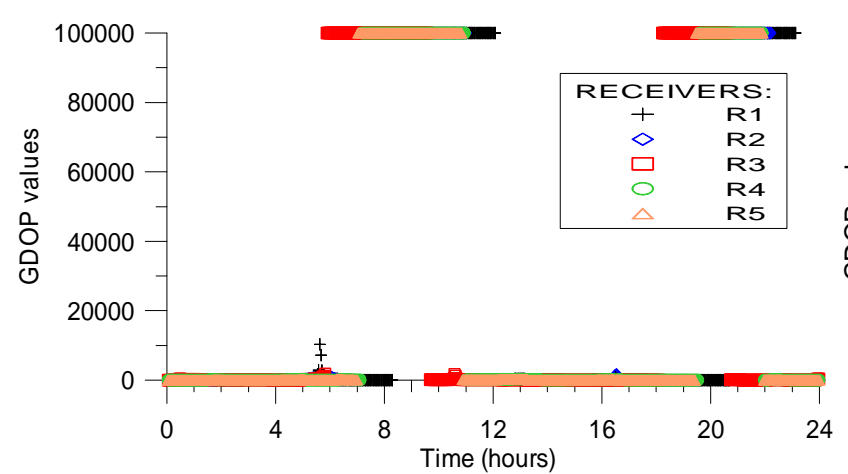

Figure 8. GDOP for Constellation 2 (10 satellites)

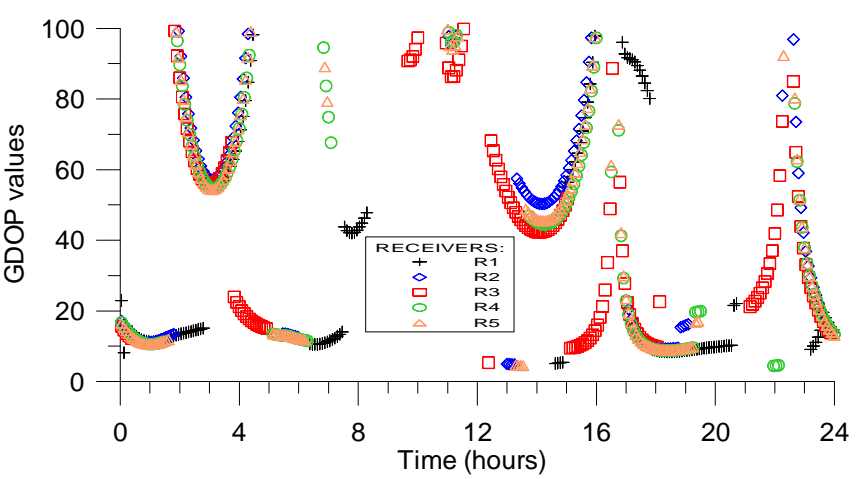

Figure 9. Zoom of Fig. 8.

Figures 8 and 9 show that with 10 satellites there are huge gaps in the coverage for the receivers, with the main one occurring around $9 \mathrm{~h}$ and when there are less then 4 satellites visible to all the receivers simultaneously. Figure 10 also shows that even when there is 4 satellites visible to the receivers the GDOP values for all the receivers are not good $(>10)$ quite frequently. The percentages of the time interval on which GDOP was less than 10 were $\{12.5 \% ; 5.4 \% ; 2.4 \% ; 7.5 \%$; and $8.2 \%\}$ for the receivers $\{\mathrm{R} 1, \mathrm{R} 2, \mathrm{R} 3, \mathrm{R} 4$, and $\mathrm{R} 5\}$ respectively.

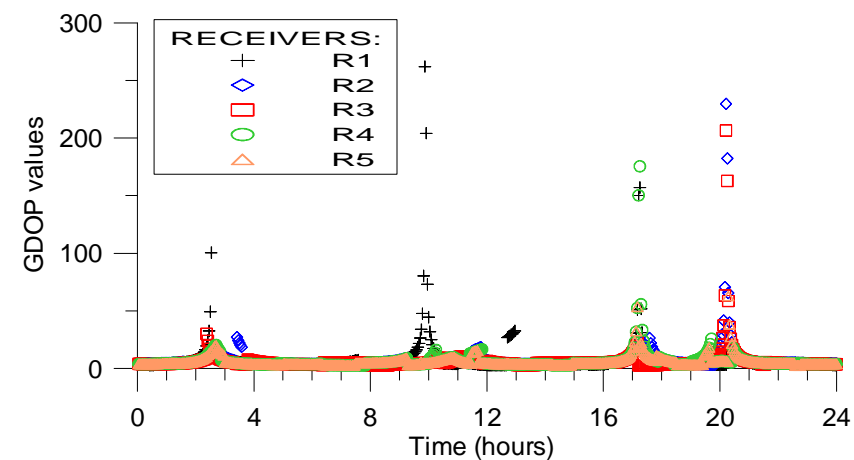

Figure 10. GDOP for Constellation 2 (12 satellites)

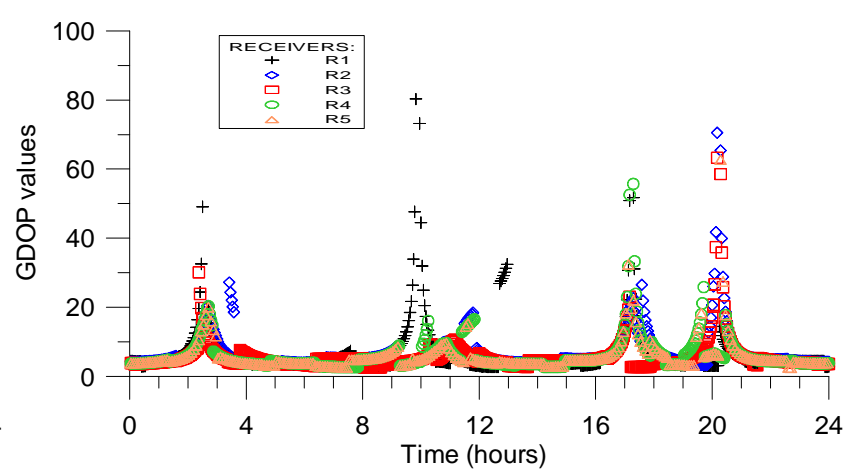

Figure 11. Zoom of Fig. 10.

Figures 10 and 11 show that with 12 satellites there are no gaps in the coverage for the receivers, but there are four big peaks in the GDOP values, affecting some receivers more than others. Figure 11 also shows that, during the peaks, the time interval where the GDOP is greater than 10 lasts around one hour or less, with the possible exception of the peak occurred at $20 \mathrm{~h}$. The percentages of the time interval on which GDOP was less than 10 were $\{87.5 \% ; 86.6 \% ; 93.4 \% ; 89.9 \%$; and $90.8 \%\}$ for the receivers $\{\mathrm{R} 1, \mathrm{R} 2, \mathrm{R} 3, \mathrm{R} 4$, and $\mathrm{R} 5\}$ respectively. 


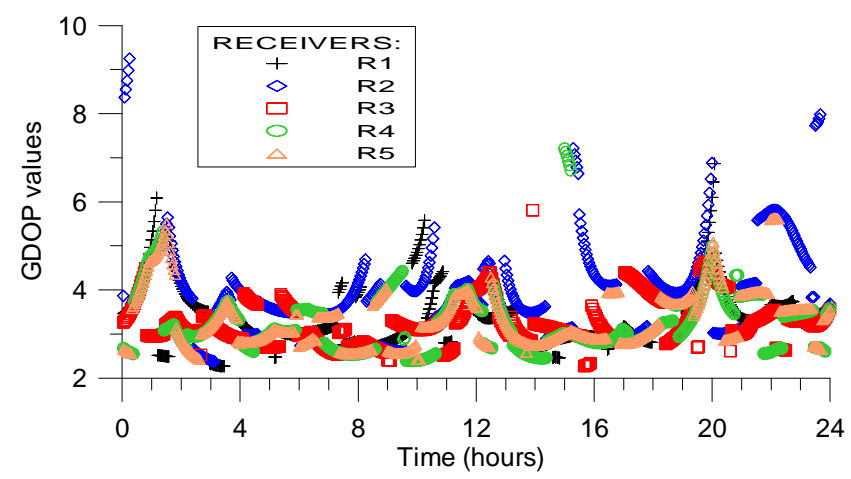

Figure 12. GDOP for Constellation 2 (14 satellites)

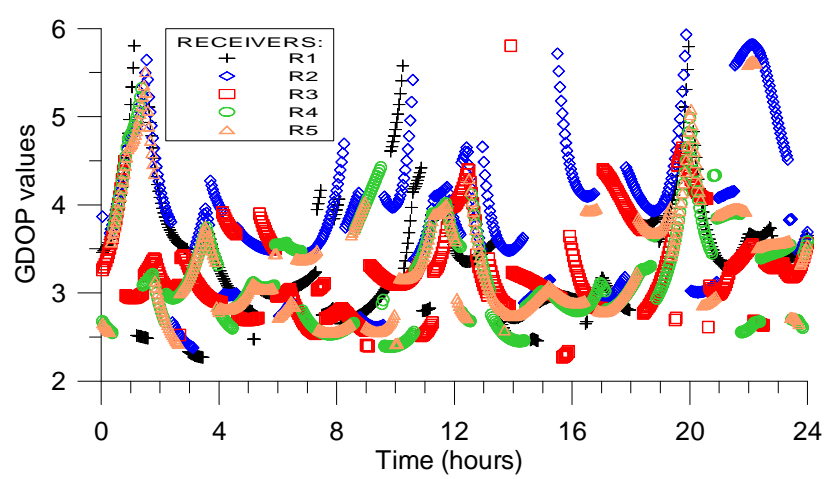

Figure 13. Zoom of Fig. 12.

Figures 12 and 13 show that with 14 satellites the coverage is very good for all the receivers during the whole time, remaining below the value of 10 all the time and below 5 most of the time. The percentages of the time interval on which GDOP is less than 10 were $100.0 \%$ for all receivers. The percentages for GDOP < 5 were $\{96.9 \% ; 87.3 \%$; 99.8\%; 98.3\%; and 97.1\% \} for the receivers $\{\mathrm{R} 1$, R2, R3, R4, and R5 respectively. In terms of computational time for Constellation 2, they were $0.15,0.31$, and 0.41 day for 10,12 and 14 satellites, respectively, on a Intel Core 2 Quad $2.83 \mathrm{GHz}$ PC with 2GB RAM.

\section{3. - Results for Constellation 3 (Geosynchronous Orbit - 35,786km)}

The best solution found for Constellation 3 is given in the Table 2, with all values in degrees.

Table 2. - Constellation 3 solutions ( 4 satellites, GDOP $=7.71$ )

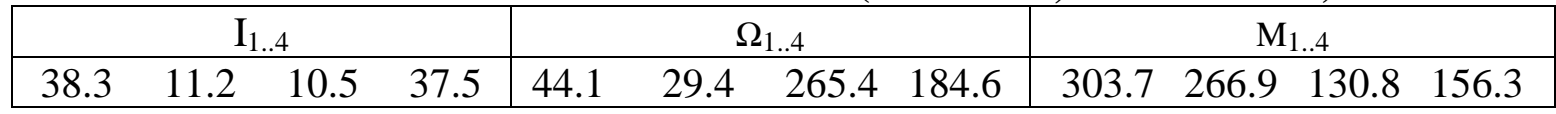

Figures 14 and 15 give the GDOP values occurred for the 5 receivers during the propagation interval with the solution given in Table 2.

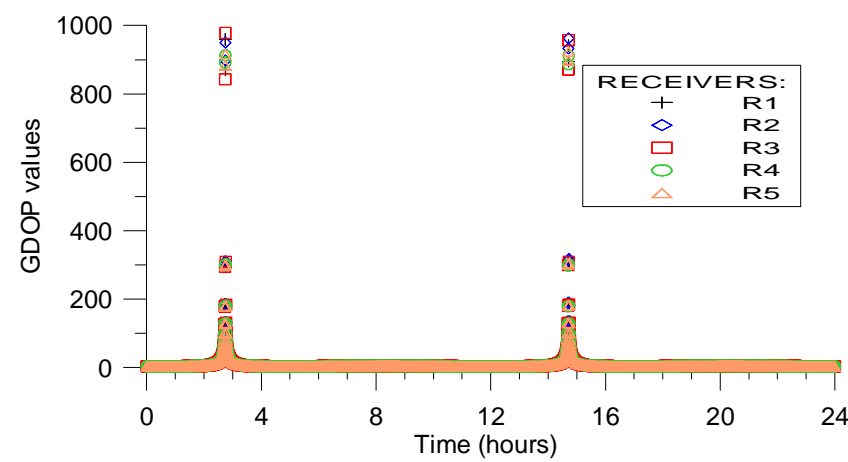

Figure 14. GDOP for Constellation 3 (4 satellites)

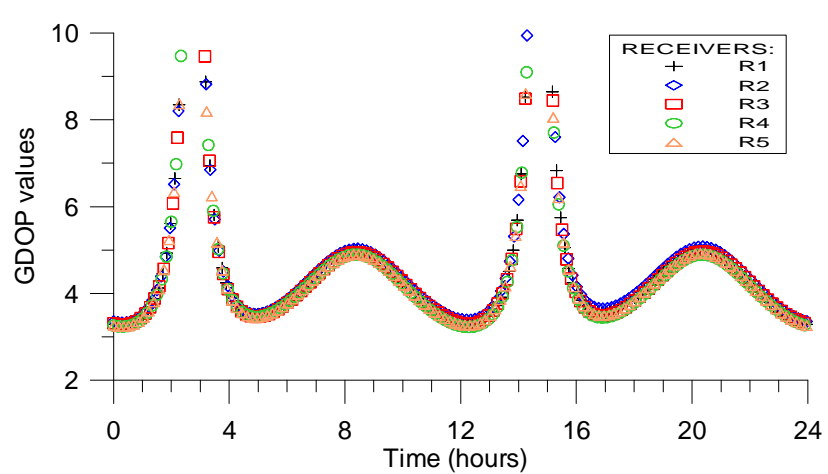

Figure 15. Zoom of Fig. 14.

As can be seen from Fig. 14, there are two big peaks in the GDOP values, equally affecting all five receivers and with a separation time of 12 hours. By its turn, Fig. 15 shows that, during the peaks, the time interval where the GDOP is greater than 10 lasts less than one hour $(\sim 47 \mathrm{~min}$ and $\sim 49 \mathrm{~min}$, via file check). The time percentages for GDOP < 10 were $\{93.4 \%$; 93.3\%; 93.5\%; 93.5\%; and 93.5\% \} for the receivers $\{\mathrm{R} 1, \mathrm{R} 2, \mathrm{R} 3, \mathrm{R} 4$, and $\mathrm{R} 5\}$ respectively. It is possible to observe that there is uniformity in these percentages, all remaining close to $93.5 \%$. Regarding the two peaks in the GDOP values occurring approximately at hour 02:45 and 14:45 of the period considered in the simulation, it was possible, with the help of the STK software, to visualize that they represent the two moments in which all satellites almost align themselves into one plane, what is a poor geometry for positioning calculations and having, as a consequence, high GDOP values. These moments are 
given in the Fig.s 16 and 17 on the following. These figures also show the orbit ground track of the four satellites, labeled as S1 to S4, and the visibility trace of the receiver R2 for The Medium Earth Orbit satellites. It is possible to observe in the figures that the visibility reach of R2 was an active bound for the optimization process, since satellites 1 and 4 have their inclinations in the very limit of the reach of R2. In terms of computational time for Constellation 3, it was 0.09 day using the hardware already mentioned.

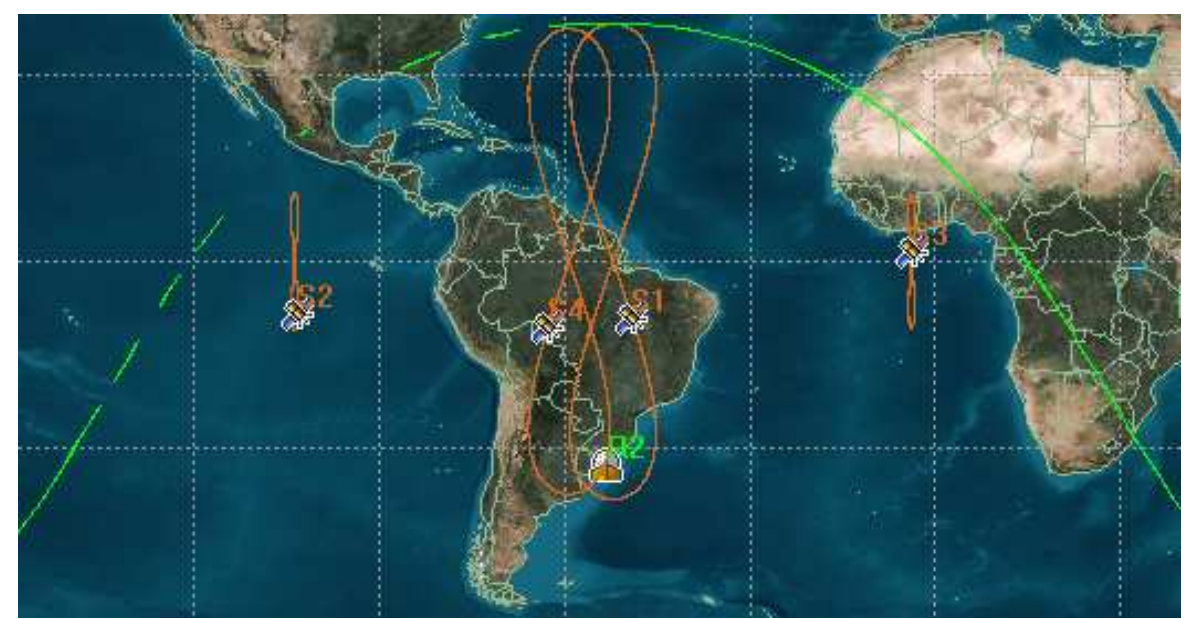

Figure 16. GDOP peak at hour 02:45

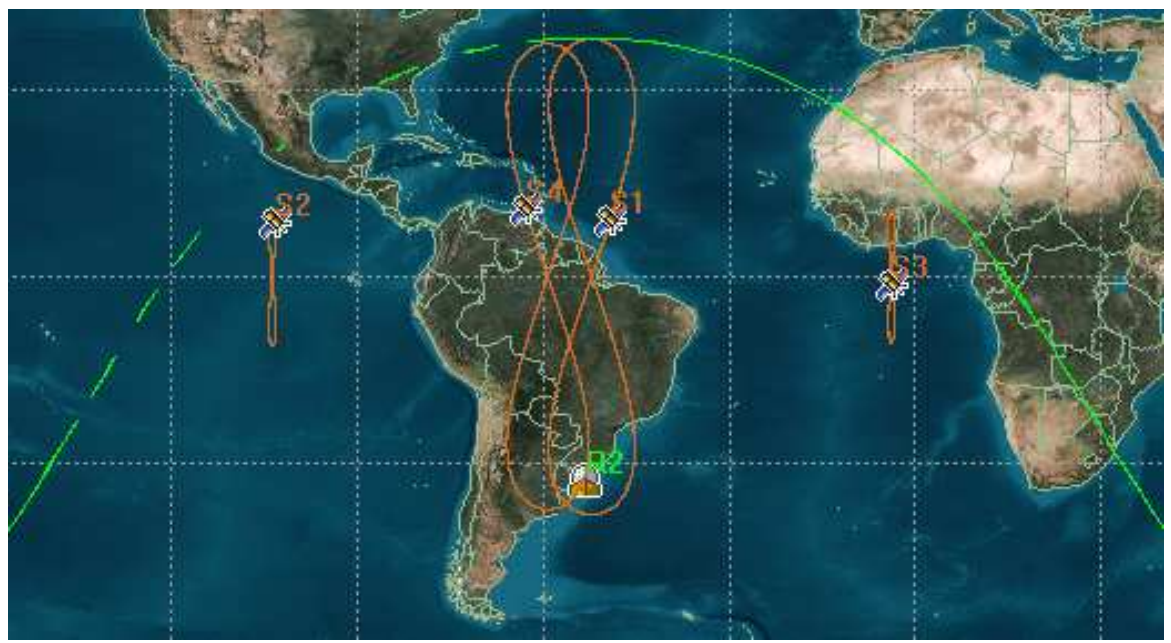

Figure 17. GDOP peak at hour 14:45

\section{Conclusions}

In this work, GDOP was used as a metric for the optimized preliminary design of three types of satellite constellations with the purpose of establishing a positioning system over the Brazilian territory. The optimization task was performed by the hybrid evolutionary algorithm GEO + ES. The results have shown that for Low Earth Orbit, more than 120 satellites are needed in order to have good coverage of the entire Brazilian territory. In the case of Medium Earth Orbit, 12 satellites presented good performance and 14 satellites presented excellent performance. In the case of the 4 geosynchronous satellites the results were as good as for 12 satellites in Medium Earth Orbit, when one uses the GDOP averages found for both at the end of the optimization process as a comparison metric. For future works, it will be interesting to see if the addition of a fifth satellite to the geosynchronous constellation would eliminate the two GDOP peaks showed with four satellites and also to find out what is the minimum number of Low Earth Orbit satellites for good coverage. Additionally, it is important to perform a full scale optimization process to the promising configurations found here in order to verify and possible improve their optimality. 


\section{Acknowledgment}

The author acknowledges and thank Dr. Valcir Orlando for his contribution during some phases of this work.

\section{References}

[1] GPS World Magazine, GPS References, http://www.gpsworld.com/gps/gps-references-6438, 2011.

[2] Wikipedia, Global Positioning System, http://en.wikipedia.org/wiki/Global_Positioning_System, 2011.

[3] Wikipedia, Global Positioning System, http://en.wikipedia.org/wiki/GLONASS, 2011.

[4] GPS World Magazine, The System, http://www.gpsworld.com/listing/122/the-system, 2011.

[5] Ma, D.-M., "Regional positioning system using low earth orbit constellations", Acta Astronautica, Vol. 58, Issue 8, pp. 387-394, April 2006.

[6] Wikipedia, Dilution of precision http://en.wikipedia.org/wiki/Dilution_of_precision_(GPS), 2011.

[7] Galski, R.L., "Development of Improved, Hybrid, Parallel, and Multiobjective versions of the Generalized Extremal Optimization Method and its application to the Design of Spatial Systems" D.Sc. Thesis (In Portuguese), Instituto Nacional de Pequisas Espaciais, Brazil, 2006. 279 p. INPE14795-TDI/1238.

[8] De Sousa, F.L., Ramos, F.M., Paglione, P., and Girardi, R.M. "New stochastic algorithm for design optimization", AIAA Journal 41 (Number 9), pp. 1808-1818, 2003.

[9] Eiben, A.E. and Smith, J.E., "Introduction to evolutionary computing", Springer-Verlag, Berlin, Germany, 2003.

[10] Bak, P. and Sneppen, K., "Punctuated equilibrium and criticality in a simple model of evolution”, Physical Review Letters, Vol. 71 (n. 24), pp. 4083-4086, 1993.

[11] Muraoka I., Galski R.L., de Sousa F.L., and Ramos F.M., "Stochastic Spacecraft Thermal Design With Low Computational Cost", Journal of Spacecraft and Rockets, Vol. 43 (n. 6), pp. 1248-1257, 2006. (ISSN 1533-6794)

[12] Vlassov V.V., de Sousa F.L., and Takahashi W.K., "Comprehensive Optimization of a Heat Pipe Radiator Assembly Filled with Ammonia or Acetone", International Journal of Heat and Mass Transfer, Vol. 49, pp. 4584-4595, 2006.

[13] De Sousa F.L., Muraoka I., and Galski R.L. "On The Optimal Positioning of Electronic Equipment In Space Platforms", Proceedings of the 19th International Congress of Mechanical Engineering (CDROM), COBEM 2007, 5-9 November, Brasília, Brazil, 2007.

[14] Analytical Graphics Inc., Satellite Tool Kit (STK), http://www.agi.com/products/by-producttype/applications/stk/, 2011. 